\title{
Minimal short-term effect of dietary 2'-fucosyllactose on bacterial colonisation, intestinal function and necrotising enterocolitis in preterm pigs
}

\author{
Malene S. Cilieborg ${ }^{1,2}$, Stine B. Bering ${ }^{1}$, Mette V. Østergaard ${ }^{1}$, Michael L. Jensen ${ }^{1}$, Lukasz Krych $^{3}$, \\ David S. Newburg ${ }^{4}$ and Per T. Sangild ${ }^{1 *}$ \\ ${ }^{1}$ Department of Veterinary Clinical and Animal Sciences, Comparative Pediatrics and Nutrition, University of Copenhagen, \\ Dyrlaegevej 68, 1870 Frederiksberg C, Denmark \\ ${ }^{2}$ National Veterinary Institute, Technical University of Denmark, Bülowsvej 27, 1870 Frederiksberg C, Denmark \\ ${ }^{3}$ Department of Food Science, University of Copenhagen, Rolighedsvej 26, 1870 Frederiksberg C, Denmark \\ ${ }^{4}$ Department of Biology, Boston College, 140 Commonwealth Avenue, 02467 Boston, MA, USA
}

(Submitted 11 April 2016 - Final revision received 6 June 2016 - Accepted 9 June 2016 - First published online 25 July 2016)

\section{Abstract}

Human milk decreases the risk of necrotising enterocolitis (NEC), a severe gastrointestinal disease that occurs in 5-10\% of preterm infants. The prebiotic and immune-modulatory effects of milk oligosaccharides may contribute to this protection. Preterm pigs were used to test whether infant formula enriched with $\alpha 1,2$-fucosyllactose (2'-FL, the most abundant oligosaccharide in human milk) would benefit gut microbial colonisation and NEC resistance after preterm birth. Caesarean-delivered preterm pigs were fed formula (Controls, $n$ 17) or formula with $5 \mathrm{~g} / 1$ 2'-FL (2'-FL, $n$ 16) for $5 \mathrm{~d}$; eight 2'-FL pigs ( $50 \%)$ and twelve Controls ( $71 \%)$ developed NEC, with no difference in lesion scores $(P=0 \cdot 35)$; 2 '-FL pigs tended to have less anaerobic bacteria in caecal contents $(P=0 \cdot 22)$, but no difference in gut microbiota between groups were observed by fluorescence in situ hybridisation and 454 pyrosequencing. Abundant $\alpha 1,2$-fucose was detected in the intestine with no difference between groups, and intestinal structure (villus height, permeability) and digestive function (hexose absorption, brush border enzyme activities) were not affected by 2'-FL. Formula enrichment with 2'-FL does not affect gut microbiology, digestive function or NEC sensitivity in pigs within the first few days after preterm birth. Milk 2'-FL may not be critical in the immediate postnatal period of preterm neonates when gut colonisation and intestinal immunity are still immature.

Key words: $\boldsymbol{\alpha 1 , 2 - F u c o s y l l a c t o s e : ~ B a c t e r i a l ~ c o l o n i s a t i o n : ~ N e c r o t i s i n g ~ e n t e r o c o l i t i s : ~ P r e t e r m ~ p i g s ~}$

Preterm birth is associated with immature organ functions and greater risk of postnatal morbidities such as necrotising enterocolitis (NEC), a life-threatening intestinal disease that occurs in $5-10 \%$ of preterm infants. The risk of NEC increases when infants are fed a milk replacer instead of human milk ${ }^{(1)}$, probably because human milk contains growth-promoting, immune-stimulating and antimicrobial components. Among these, human milk oligosaccharides (HMO) are fucosylated and sialylated carbohydrate structures that are speculated to modulate mucosal immunity, growth and bacterial colonisation ${ }^{(2)}$. With concentrations of upto $23 \mathrm{~g} / \mathrm{l}^{(3)}$, HMO represent the third most-abundant group of solids in human milk ${ }^{(4)}$, but they are almost absent in cow's milk ${ }^{(2)}$. HMO are largely indigestible ${ }^{(5)}$ but may have important intestinal luminal functions. Among the many different HMO, $\alpha 1$,2-fucosyllactose (2'-FL) is among the most abundant $(2.5 \mathrm{~g} / 1)^{(6,7)}$, and it has been shown to increase the growth of Bifidobacteria, while suppressing Clostridia sp. and Enterobacteria sp. ${ }^{(8,9)}$. Further, 2'-FL shares structural homology with fucosylated $\mathrm{H}$-antigens on the intestinal epithelium to which various pathogenic bacteria adhere, including Escherichia coli F18 that is pathogenic to weanling pigs ${ }^{(10,11)}$. 2'-FL decreases adhesion of pathogenic bacteria in vitro ${ }^{(12-14)}$, and $2^{\prime}$-FL levels in human milk are inversely related to the incidence of infectious diarrhoea with Campylobacter in infants ${ }^{(15)}$.

NEC is often associated with overgrowth of potentially harmful bacteria such as Clostridium sp., Klebsiella sp., E. coli, Pseudomonas sp. and Staphylococcus sp. ${ }^{(16)}$. Potentially, 2'-FL can reduce the risk of NEC by acting in parallel with intestinal fucosylated glycans to improve gut colonisation via prebiotic actions and inhibition of specific harmful bacteria. Preterm infants that do not express $\mathrm{H}$-antigen show increased NEC and death rates, indicating the importance of intestinal glycan fucosylation $^{(17)}$. 2'-FL may also modulate immune signalling by the gut mucosa, as demonstrated for sialylated $\mathrm{HMO}^{(2)}$. Immune modulation can explain the protection against NEC-like lesions and increased overall survival of neonatal rats after supplementation with a sialylated $\mathrm{HMO}^{(18)}$, the same $\mathrm{HMO}$ associated

Abbreviations: FISH, fluorescence in situ hybridisation; HMO, human milk oligosaccharides; OUT, Operational Taxonomic Unit.

* Corresponding author: P. T. Sangild, fax +45 3533 2469, email pts@sund.ku.dk 
with decreased NEC incidence in infants from HIV-infected mothers ${ }^{(19)}$. HMO, including $2^{\prime}$-FL, may increase intestinal maturation $^{(20)}$ and modulate immune signalling pathways ${ }^{(21)}$, in part via inhibiting the CD14-mediated, endotoxin-induced inflammation $^{(22)}$.

Preterm milk contains particularly high levels of 2'-FL $(7 \mathrm{~g} / \mathrm{l})^{(3)}$, suggesting a protective role in preterm infants. We tested the hypothesis that dietary supplementation with physiological doses $(5 \mathrm{~g} / \mathrm{l})$ of $2^{\prime}$-FL would decrease NEC incidence and intestinal lesions by modulating bacterial colonisation and stimulating mucosal growth and digestive function in newborn preterm pigs. Preterm pigs show a high incidence of NEC-like lesions when fed increasing amounts of enteral milk diets, especially formula, during the first 1-2 weeks after birth. Intestinal structure and function were investigated on day 5 , after a relatively fast transition from parenteral to enteral feeding. Hence, the present study focused on the effects of a 2'-FL-enriched formula on the immature intestine during the immediate postnatal period.

\section{Methods \\ Animal protocol and experimental design}

In total, thirty-three pigs from two sows (Large White $\times$ Danish Landrace) were delivered by caesarean section at $90 \%$ gestation and reared individually in temperature- and $\mathrm{O}_{2}$-regulated incubators. While they still remained sedated after the caesarean section, the pigs were fitted with umbilical (4F; Portex) and orogastric catheters (6F; Pharmaplast). Mother's serum $(12 \mathrm{ml} / \mathrm{kg}$ during the first $24 \mathrm{~h}$ ) was provided via the umbilical catheter for passive immunisation. For $2 \mathrm{~d}$, the pigs received parenteral nutrition (PN, 4-6 ml/kg per h, Nutriflex lipid plus; Braun) with added amino acids (Vamin; Fresenius Kabi). As a supplement to PN, minimal enteral nutrition (MEN) with the respective enteral diets was provided for the first $2 \mathrm{~d}$ $(2-3 \mathrm{ml} / \mathrm{kg}$ per $3 \mathrm{~h})$. To standardise bacterial colonisation, an oral inoculation of maternal faecal bacteria $\left(2.5 \times 10^{4}\right.$ colonyforming units (cfu)) was given on day 1 . The PN-MEN period was followed by full enteral feeding with infant formula from day 3 to $5(15 \mathrm{ml} / \mathrm{kg}$ per $3 \mathrm{~h}$ of Pepdite, Maxipro and LiquigenMCT; SHS International; $4151 \mathrm{~kJ} / 1$ energy; $64 \mathrm{~g} / \mathrm{l}$ protein; $47 \mathrm{~g} / 1$ carbohydrate with $90 \%$ maltodextrin; $\left.61 \mathrm{~g} / 1 \mathrm{fat}^{(23)}\right)$. The pigs were stratified according to birth weight and sex into pigs fed Control formula ( $n$ 17) or 2'-FL formula ( $n$ 16) containing freeze-dried 2'-fucosyllactose (Glycosyn) at $5 \mathrm{~g} / \mathrm{l}$. The animal procedures followed our standard protocol ${ }^{(24)}$, and were approved by the National Committee on Animal Experimentation, Denmark, based on the ARRIVE (Animal Research: Reporting of In Vivo Experiments) Guidelines.

\section{Necrotising enterocolitis evaluation and tissue collection}

Pigs were checked every $3 \mathrm{~h}$ for NEC symptoms including bloody diarrhoea, lethargy, respiratory distress and abdominal distension, until euthanasia and tissue collection on day 5. However, pigs that developed NEC symptoms were immediately euthanised (pentobarbitone, $60 \mathrm{mg} / \mathrm{kg}$, i.v.) and tissue samples were collected. At necropsy, the stomach, proximal, middle and distal regions of the small intestine, and the colon were scored for NEC lesions according to our standard macroscopic evaluation system: $1=$ normal, $2=$ local hyperaemia and oedema, 3 =hyperaemia, extensive oedema and local haemorrhage, $4=$ extensive haemorrhage, 5=local necrosis and pneumatosis intestinalis and $6=$ extensive necrosis and pneumatosis intestinalis. NEC was defined as a score of at least three in at least one gastrointestinal region ${ }^{(24)}$. Organs were weighed, and tissues from the three small intestinal regions were collected and snap-frozen for later analyses; three sections equally dispersed along the small intestine (proximal, middle and distal intestine at 17, 50 and 83\% from the stomach) were formalin fixed and paraffin embedded for histology, fluorescence in situ hybridisation (FISH) and lectin hybridisation.

\section{Digestive function, mucosal integrity and intestinal fucosylation}

Galactose absorption was measured in pigs before transition to full enteral feeding using blood samples collected $20 \mathrm{~min}$ after the administration of an oral bolus of $15 \mathrm{ml} / \mathrm{kg}$ of $5 \%$ galactose and $2 \%$ mannitol $^{(25)}$. The combined lactose digestive and absorptive capacity was measured in pigs $36 \mathrm{~h}$ after transition to full enteral feeding as plasma galactose $30 \mathrm{~min}$ after a $15 \mathrm{ml} / \mathrm{kg}$ bolus of $15 \%$ lactose. Intestinal permeability was assessed as the postmortem urine ratio of lactulose:mannitol ${ }^{(26)}$ after oral administration of $0.5 \mathrm{~g} / \mathrm{kg}$ lactulose and $0.3 \mathrm{~g} / \mathrm{kg}$ mannitol $4 \mathrm{~h}$ before euthanasia. The above-mentioned analyses were performed for all pigs, whereas the tissue analyses were performed only for twenty-one pigs from one litter ( $n 10$ and 11 for 2'-FL and Control pigs, respectively).

Mucosal disaccharidase activities (maltase, sucrase, lactase) and peptidase activities (aminopeptidase $\mathrm{N}$ and $\mathrm{A}$ (ApN and ApA) and dipeptidyl peptidase IV (DPPIV)) were measured in tissue homogenates of the three regions of the small intestine $^{(27)}$. Mucosa was scraped from $10-\mathrm{cm}$ fresh sections of the proximal, middle or distal small intestine to determine dry weight proportions. Villus height was measured by scanning pictures of paraffin-embedded sections of the distal small intestine (ArrayWoRx microarray scanner; Applied Precision) using ImageJ version 1.44 (US National Institutes of Health).

Intestinal $\alpha 1,2$-fucosylation was measured on paraffinembedded cross-sections $(3 \mu \mathrm{m})$ from the proximal, middle and distal small intestine by hybridisation with $\alpha 1,2$-fucose-specific lectin, Ulex Europaeus Agglutinin I (UEA-I; Vector Laboratories), labelled with green fluorescein isothiocyanate. Sections were evaluated using an Axioimager M1 epifluorescence microscope (Carl Zeiss). Sections were scored for $\alpha 1,2$-fucose binding on the basis of a green fluorescence signal, where $1=$ none, $2=\mathrm{a}$ few positively stained goblet cells, $3=$ several positively stained goblet cells, mainly in the crypt area, or moderate luminal green fluorescence, $4=$ abundant, positively stained goblet cells along the entire mucosal lining and $5=$ abundant, positively stained goblet cells along the entire mucosal lining together with extensive luminal fluorescence. 


\section{Microbiology}

Caecal contents were plated on calf blood agar (SSI Diagnostica), cultivated overnight under anaerobic conditions and total anaerobic bacteria was enumerated. Microbial compositions of caecal contents and of the mucosa of the distal small intestine were analysed by 454 pyrosequencing. Samples were homogenised by bead beating for $2 \mathrm{~min} / 20 \mathrm{~Hz}$ in a tissue lyser (Qiagen). After DNA purification using the Maxwell platform (Promega), the V4-V5 region of the bacterial $16 \mathrm{~s}$ rDNA was amplified using bar-coded primers $(519 \mathrm{~F}$ 5'-CAGCAGCCGCGGTAATAC-3'，926R 5'-CCGTCAATTCCTTT GAGTTT-3'). DNA quantity and quality were analysed in a Agilent 2100 Bioanalyzer (Agilent Technologies) using the Agilent RNA 1000 Nano Kit, and 50 ng DNA from each of the thirty-nine samples (twenty-one from small intestinal tissues and eighteen from caecal contents) were pooled for sequencing on a 454 Roche GS FLX titanium platform at LGC Genomics following the manufacturer's protocols (Roche). The data set was analysed in the Quantitative Insight Into Microbial Ecology (QIIME) open-source package (version 1.7.0). Raw data underwent quality control, de-noising, chimera filtering and Operational Taxonomic Unit (OTU) picking ${ }^{(28)}$, and clustering at $97 \%$ relatedness using UCLAST. Representative sequences from each cluster were aligned with pyNAST and subjected to the Ribosomal Database Project. Alpha diversity was calculated, as observed species and Chao1 and Shannon indices for OTU tables were unified to 2000 sequences per sample. Principal coordinate analysis (PCOA) plots were generated with the Jackknifed Beta Diversity workflow-based ten subsampled OTU tables and $85 \%$ of sequences taken for each jackknifed subset.

Bacterial abundance in situ was evaluated using the same tissues as above after hybridisation with Alexa fluor 555-labelled oligonucleotide probes (Eurofins MWG Operon) targeting general bacterial 16S rRNA (5'-GCTGCCTCCC GTAGGAGT-3') ${ }^{(29)}$. Bacterial micro-colonies were visualised by scanning, and sections were given a FISH-score based on the red fluorescence signal: $1=$ no/very few bacteria, $2=a$ few bacteria spread in the intestinal lumen or tissue, $3=$ several colonies in the intestinal lumen or tissue and $4=$ extensive and abundant bacteria in the intestinal lumen and/or tissue. Selected tissue sections were further analysed by confocal microscopy (Zeiss LSM 710; Carl Zeiss microimaging) for detailed associations between colonising bacteria and intestinal mucosa.

\section{Calculations and statistical analyses}

Sample size for the study population was determined on the basis of a power calculation using the estimated means and standard deviations for NEC incidence across sixteen previous preterm pig studies (69 (SEM 21)\%, $n$ 210). In these studies, the most effective diets were interventions with lactose ${ }^{(30)}$, colostrum $^{(24)}$ and oral antibiotics ${ }^{(31)}$ that reduced the basic NEC rates by $70-90 \%$. On the basis of this background, an expected effect of HMO supplementation was set to a reduction in the NEC rate by $30 \%$. Consequently, the required sample size for $80 \%$ power was seventeen. Differences in NEC incidence were tested between treatment groups using Fisher's exact test. Other data were analysed using R version 3.0.1. Differences in weight gain, NEC scores, sugar absorption, intestinal permeability and mucosal proportions were tested using the lmer function of linear mixed model analysis with sow as the random variable and with treatment, birth weight and sex as fixed variables. After model reduction, additionally measured parameters were compared by two-way ANOVA to evaluate the treatment effect. The $l m$ function was used to test villus height, enzyme activity, cfu counts and FISH, with treatment, birth weight and sex as fixed variables. For pyrosequencing data, group differences in PCOA plots were evaluated by analysis of similarities (QIIME) using weighted and unweighted uniFrac distance metrics generated from rarefied OTU tables (excluding seven samples with $<2000$ reads). A $t$ test (Monte Carlo, 999 permutations, QIIME) was used to calculate differences in alpha diversity. Differences in the five, most-dominant OTU were tested using the Imer function in R, where treatment, birth weight and sex were fixed variables. Normal distribution of all measured parameters was tested by plotting the residuals and by the qq-plot using the qqnorm function. All values are presented as means and standard errors, unless otherwise stated. For all analyses, $P<0.05$ was used as the critical level of significance.

\section{Results}

\section{Clinical outcomes}

Of the thirty-three preterm pigs, on day 5, eight 2'-FL pigs ( $50 \%$ ) and twelve Controls (71\%) were diagnosed with NEC lesions $(P=0 \cdot 30)$. The average lesion scores in the small intestine were 1.5 (SEM 0.1) v. 1.6 (SEM 0.1) for 2'-FL pigs and Controls, respectively $(P=0.77)$, and the average colon lesion scores were 1.9 (SEM $0 \cdot 3$ ) v. $2 \cdot 3$ (SEM $0 \cdot 3$ ), respectively $(P=0 \cdot 35$, Fig. 1 ). There were no differences between groups (means across treatments, $P=0 \cdot 15-0.80$ ) for birth weight (908 (sEM 38) g), relative weight gain $(-0.62(\operatorname{SEM} 17 \cdot 8) \mathrm{g} / \mathrm{kg})$ and relative weights $(\mathrm{g} / \mathrm{kg})$ for stomach (7.6 (SEM 0.3)), small intestine (37.2 (SEM 1.3)), colon (11.4 (SEM 0.6)), heart (7.9 (SEM 0.3)), lungs (25.8 (SEM 1.3)), liver (28.4 (SEM 0.8)), kidneys (9.8 (SEM 0.2)) and spleen (1.6 (SEM 0.1)).

\section{Digestive function, mucosal integrity and fucosylation}

Supplementation with 2'-FL did not influence the initial galactose absorptive capacity on day 3 (499 (SEM 79) $\mu \mathrm{mol} / \mathrm{l}$ across

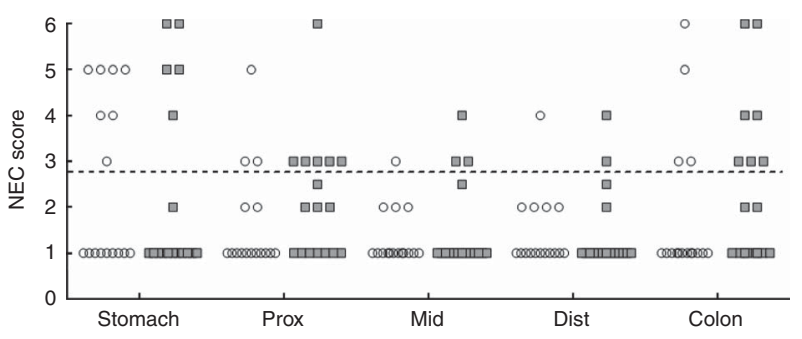

Fig. 1. Necrotising enterocolitis (NEC) scores in five gastrointestinal tract regions shown as a scatter plot where each dot within a region represents one pig. NEC is defined as a score of at least three in at least one gastrointestinal region (-...-.-.). There were no differences between $a 1,2$-fucosyllactose $(2 '-F L, O)$ and Control pigs $(\square)$. Prox, proximal small intestine; mid, middle small intestine; dist, distal small intestine. 

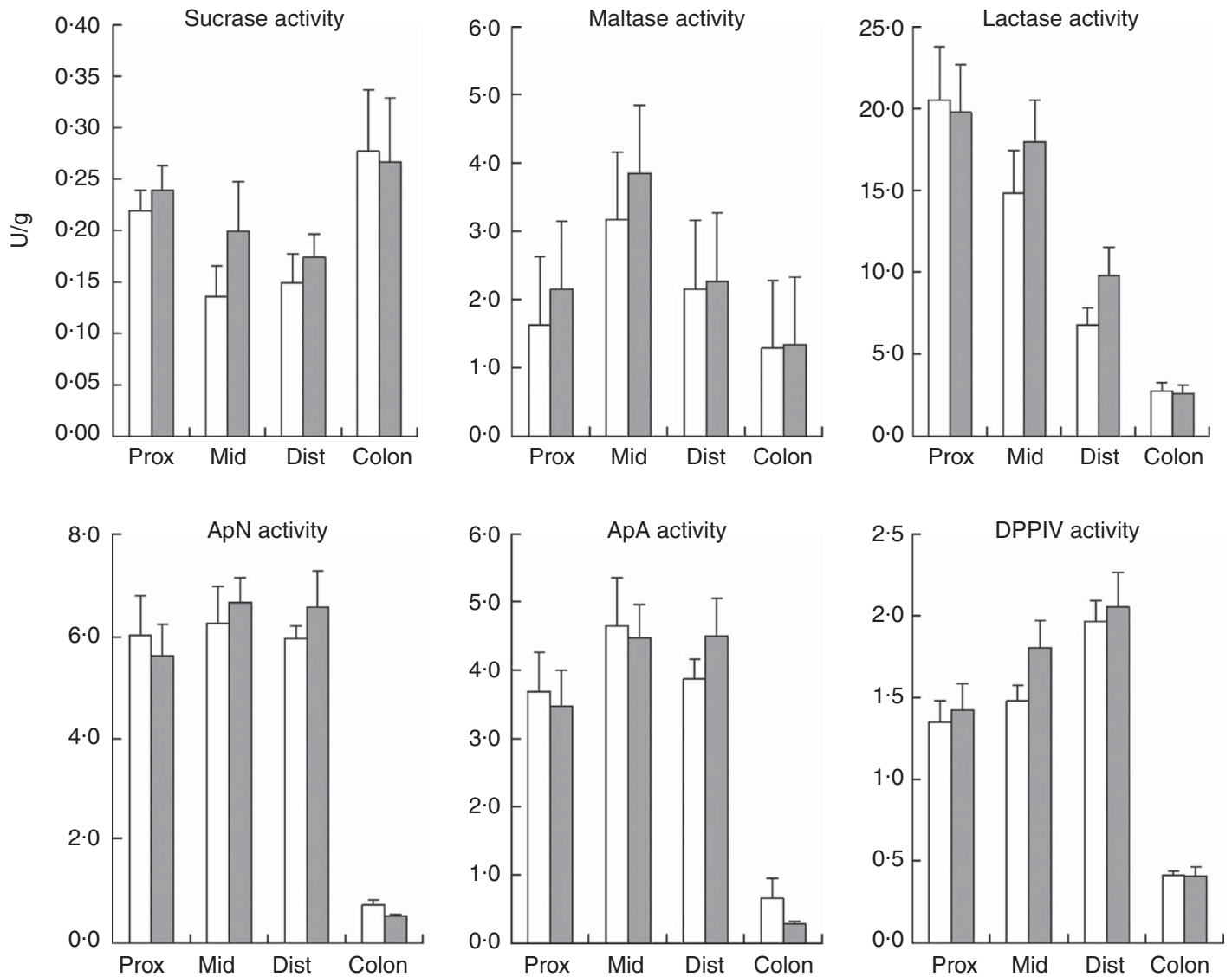

Fig. 2. Brush-border enzyme activity (values are means, with their standard errors) for sucrase, maltase, lactase, aminopeptidase $N(A p N)$, aminopeptidase $A$ (ApA) and dipeptidyl peptidase IV (DPPIV). There were no differences between $a 1,2$-fucosyllactose (2'-FL, $\square$ ) and Control pigs ( $\square$ ). Prox, proximal small intestine; mid, middle small intestine; dist, distal small intestine.

groups, $P=0.66$ ) or on day 5 (39.8 (sem 9.6), $P=0.70)$. Likewise, the activities of six brush border enzymes (U/g) were not affected by 2'-FL supplementation (Fig. 2), neither in the small intestine (sucrase: 0.19 (SEM 0.01), maltase: 2.55 (SEM 0.25), lactase: 15.01 (SEM 1.41), ApN: 6.19 (SEM 0.33), ApA: 4.11 (SEM 0.31), DPPIV: 1.68 (SEM 0.07), $P=0 \cdot 13-0.90$ between groups) nor in the colon (sucrase: $0 \cdot 27$ (SEm 0.04), maltase: $1 \cdot 31$ (sEm 0.08), lactase: $2 \cdot 68$ (SEM 0.37), ApN: 0.65 (sEm 0.06), ApA: 0.47 (sem 0.15), DPPIV: 0.41 (sEm 0.03), $P=0.07-0.91$ between groups). Similarly, there was no effect of 2 '-FL on the lactulose: mannitol ratio $(0.12$ (SEM 0.03$), P=0.56$ between groups), mucosal proportion (71 (SEM 1 ) \% dry weight, $P=0 \cdot 80-0 \cdot 94$ ) or villus height (553 (sEm 30) $\mu \mathrm{m}, P=0 \cdot 26-0 \cdot 97$ ). In situ hybridisation with fluorescence-labelled UEA-1 revealed high levels of endogenous $\alpha 1,2$ '-fucose (Fig. 3(a) and (b)), with no difference between 2'-FL pigs and Controls (score 3.4 (SEM 0.4) v. 3.0 (sEm $0 \cdot 4$ ), respectively, $P=0 \cdot 44$ ). Goblet cells and the mucus layer were highly stained in most pigs (Fig. 3(a)), whereas in some pigs $\alpha 1,2$-fucose was found in enterocyte membranes (Fig. 3(b)).

\section{Microbiology}

Caecal content cultures showed a trend towards less abundance of anaerobic bacteria in 2'-FL pigs but without significance $\left(1 \cdot 6(\right.$ SEM $0 \cdot 6) \times 10^{10}$ v. $2.7($ SEM $\left.0 \cdot 6) \times 10^{10} \mathrm{cfu} / \mathrm{ml}, P=0 \cdot 22\right)$. FISH analyses of bacteria adhering to the intestinal mucosa revealed that the majority of pigs were densely colonised with bacteria that often appeared to have invaded the mucosa (Fig. 3(c) and (d)). However, the bacterial density was independent of treatment, and no differences in FISH-score were detected between 2'-FL pigs and Controls (2.3 (SEM 0.2) across six intestinal regions, $P=0 \cdot 40-0 \cdot 94)$.

Pyrosequencing resulted in 11804 (SEM 996) sequences per sample ( $n$ 39) with no difference detected between intestinal contents and tissues and between the two treatments $(P=0 \cdot 2$ and $0 \cdot 4$, respectively). More separate OTU were identified in tissue than in caecal contents (21.1 (SEM 2.6) v. 11.8 (SEM 0.7), respectively, $P<0.05$ ), supported by a higher alpha diversity in tissue $v$. contents as well $(P<0 \cdot 05)$. However, there was no difference in alpha diversity between 2 '-FL and Control pigs $(P=0 \cdot 83)$. Lactobacillus, Enterococcus, Turicibacter, Streptococcus and Clostridiaceae (all Firmicutes) were the five most-dominant OTU, accounting for $94 \%$ of the total sequences in intestinal tissue and $98 \%$ in caecal contents (Fig. 4(a)). The relative abundance of Lactobacillus was lower in tissue than in caecal contents $(P=0.06)$, whereas the abundance of more rare OTU was greater in tissues than in caecal contents. This was especially true for $2^{\prime} \mathrm{FL}$ pigs, where rare OTU were $0.5 \%$ in caecal contents and $8.5 \%$ in tissues, whereas in Controls these 

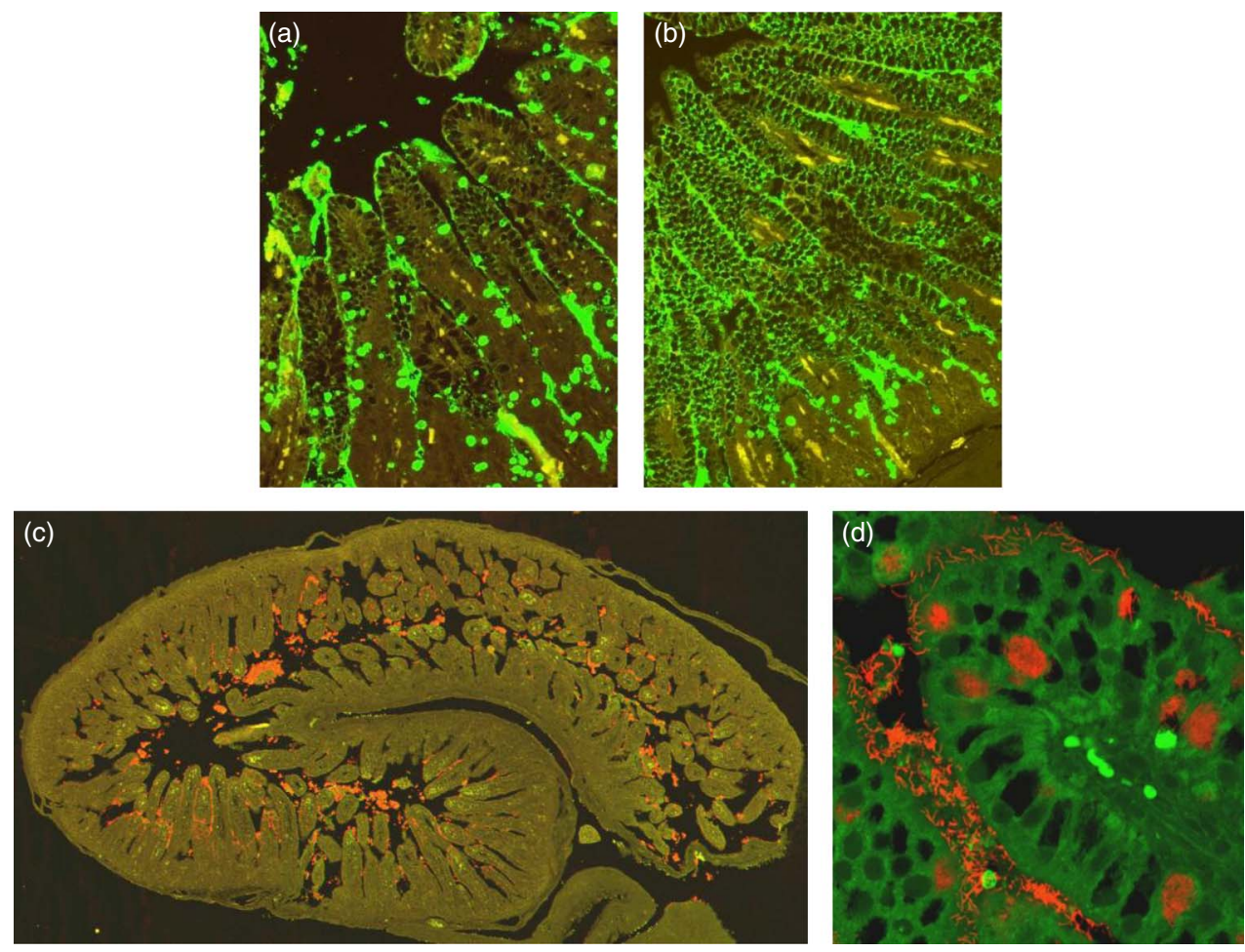

Fig. 3. Representative tissue sections of the small intestine hybridised with green fluorescein isothiocyanate-labelled lectin specific for $a 1,2$ '-fucose (a, b). Mainly goblet cells and the mucus layer were stained positive for $a 1,2$ 'fucose in both treatment groups (a), but it was also incorporated in the enterocyte membranes of some pigs (b). Representative tissue section of the small intestine hybridised with red fluorescence-labelled probes targeting 16S rRNA of general bacteria (c). Confocal microscopy reveals close association between the gut epithelium and bacteria and even bacterial invasion of the enterocytes (d).

values were $3 \cdot 2$ and $4 \cdot 2 \%$, respectively. The relative distribution of the five dominant taxa tended to differ between 2'-FL and Control pigs in both tissues and caecal contents (Fig. 4(a)), but only Enterococcus in caecal contents showed significantly different proportions between 2 '-FL and Control pigs $(P<0.05$; $P=0.12$ for tissue). PCoA plots, both weighted (quantitative) and unweighted (qualitative), did not reveal any differences between 2'-FL and Control pigs, neither for tissue nor for caecal contents $(P=0 \cdot 2-0 \cdot 5$, Fig. 4(b)).

\section{Discussion}

NEC is a life-threatening intestinal inflammatory disease in preterm infants, which is closely associated with the progression of intestinal bacterial colonisation and enteral feeding, especially using formula. Human milk is rich in HMO, whereas formula is based on cow's milk and contains limited amounts of oligosaccharides. The concentration of the most abundant HMO in human milk, 2'-FL, is particularly high following preterm birth $^{(3)}$, and this HMO has been shown to inhibit colonisation with pathogens ${ }^{(12-14,17,32)}$ and enhance the proliferation of putative beneficial bacteria such as Bifidobacteria ${ }^{(8,9)}$. In addition, 2'-FL and other HMO may exert microbiota-independent maturation of gut immunity and digestive functions ${ }^{(18,20-22)}$ and this could be particularly important for newborn preterm infants. Contrary to our hypothesis, we now show that addition of 2'-FL to formula at a dose of $5 \mathrm{~g} / 1$ has limited effects on bacterial colonisation, mucosal fucosylation, mucosal structure, digestive function and NEC sensitivity within the first $5 \mathrm{~d}$ after preterm birth in pigs. Whether 2'-FL supports intestinal maturation in the long term, when gut bacterial colonisation and intestinal immunity become more mature, remains to be investigated.

The chosen 2'-FL concentration ( $5 \mathrm{~g} / \mathrm{l})$ was within the normal range found in human milk ${ }^{(3,6)}$. A concentration of $2 \mathrm{~g} / \mathrm{l}$ was previously found to increase the growth of beneficial bacteria and to decrease the density of potentially harmful bacteria ${ }^{(9)}$. In term newborn pigs, doses upto $10 \mathrm{~g} / 1$ 2'-FL were well tolerated and a concentration of minimum $5 \mathrm{~g} / \mathrm{l}$ was required to inhibit adhesion of the porcine pathogen E. coli F18 to porcine enterocytes in vitro $^{(33)}$. To make sure that all the (initially sterile) caesarean-delivered pigs in this study were equipped with the same initial culture of maternal bacteria, we provided them with a small dose of faecal microbiota $\left(10^{4} \mathrm{cfu}\right)$ from their mother along with their first formula feed. Nevertheless, the subsequent microbiota that colonised the intestines of pigs during the following days appeared minimally affected by $5 \mathrm{~g} / \mathrm{l}$ 2 '-FL in the formula. The concentration of 2'-FL in human milk is highest in colostrum and declines thereafter ${ }^{(6)}$. Likewise, the amounts are higher in milk from mothers delivering preterm $(7 \mathrm{~g} / 1)^{(3)}$ compared with term milk $(2 \mathrm{~g} / \mathrm{l})^{(6)}$. Although this may indicate an increased need for 2'-FL just after birth, and particularly preterm birth, variation in milk constituents with gestational age and lactation length do not necessarily reflect physiological needs. In humans, secretion of 2'-FL depends on maternal genotype and about $20 \%$ of Caucasian women are 
(a)

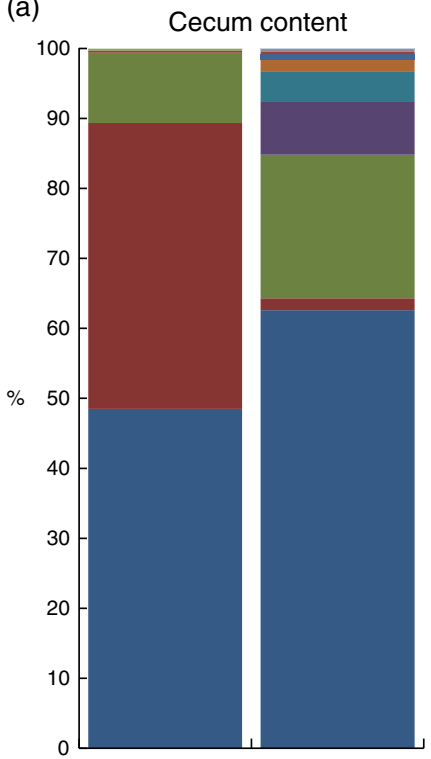

2'-FL

Control

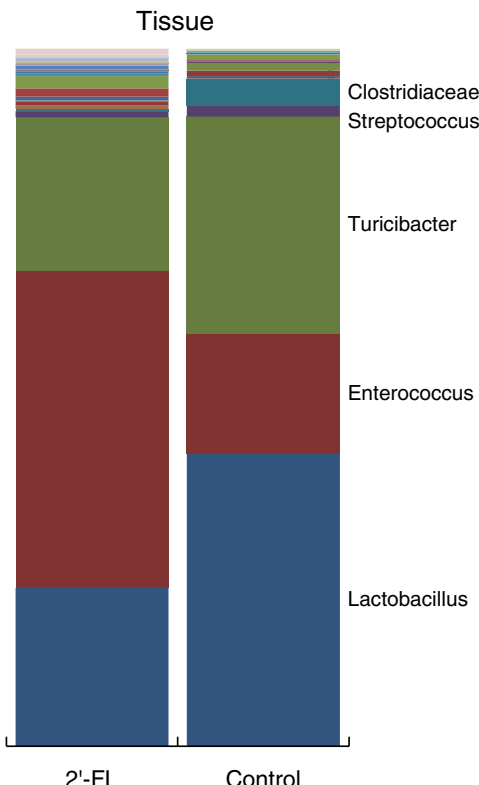

(b)

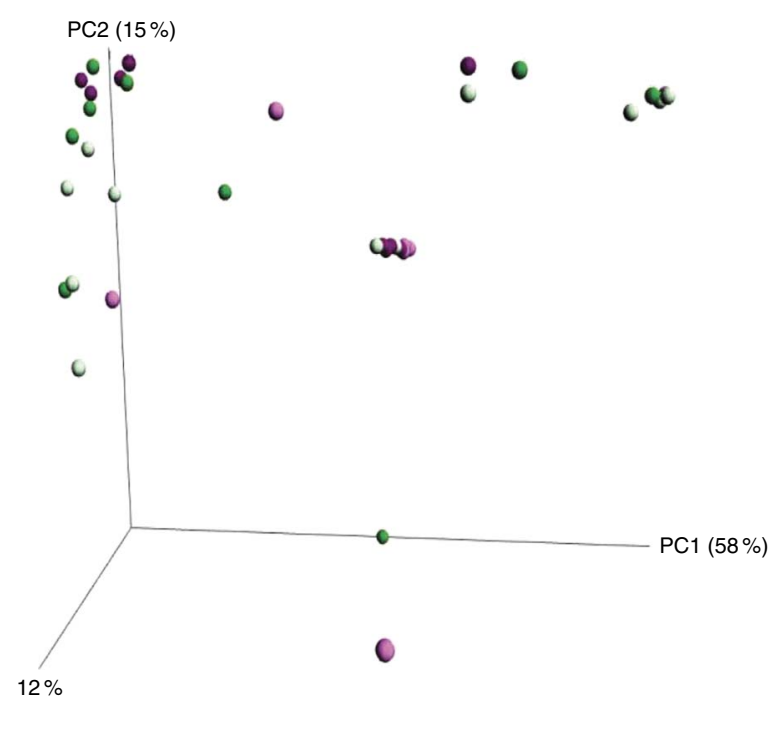

Rare OTU

- Ruminococcaceae

- Enterobacteriaceae

- Lachnospiraceae

- Leuconostoc

Flexispira coccaceae Bacteroides

- Mogibacteriaceae

- Blautia

- Staphylococcus

- Lactococcus

Bacteroidales

- Clostridiales

- Megasphaera

Megasphaera

Delftia

Ruminococcus

- Stenotrophomonas

Bacteroidales

- Paraprevotellaceae

- Campylobacter

- Bacteroidales

Coprococcus

Phascolarctobacterium Mogibacterium

- Burkholderiales

- Mogibacterium

Control

Fig. 4. Relative bacterial abundance at the genus level after 454 pyrosequencing of the caecal and tissue contents of $a 1,2-$ fucosyllactose (2'-FL) and Control pigs (a) and principal coordinate analyses (PCoA) scatter diagram (b) showing the weighted distribution of 2'FL pigs ( $\square$ ) and Control pigs ( $\square$ ). Dark colours: caecal contents; light colours: tissue contents. OUT, Operational Taxonomic Unit.

non-secretors ${ }^{(7)}$. Although infants of low-secretor mothers appear to be at higher risk of diarrhoea ${ }^{(15)}$, a relationship between NEC risk and maternal genotype or 2'-FL concentration has not been clearly established, although in one study infants with low secretor status had a 10-fold increased risk of NEC-related death and an 18-fold increased risk of death due to sepsis ${ }^{(17)}$.

In preterm pigs, we observed an abundant presence of endogenous $\alpha 1,2$ '-fucose in the intestinal mucosa, as shown by lectin hybridisation, but we did not find any relation among these levels and the degree of NEC lesions, type of microbiota or other intestinal parameters. Thus, it is possible that the beneficial effects of fucosylated compounds along the mucosal lining remain inadequate in the preterm newborn pig to protect against the pro-inflammatory effects of bacterial colonisation when fed formula devoid of the bioactive compounds of the mother's milk. Further, addition of 2'-FL did not appear to increase the tissue scores for $\alpha 1,2^{\prime}$-fucose, although there was a tendency towards decreased NEC incidence and score in the colon region. This, together with a tendency towards decreased caecal bacterial density in 2'-FL pigs, indicates a marginal beneficial effect of 2'-FL supplementation for the colon.
It is possible that such an effect would not become fully expressed until after gut bacterial colonisation becomes more established and after a longer period of 2'-FL supplementation of formula. Even though the number of pigs included is sufficient to secure appropriate power to the study, the pigs were derived from only two litters, and inclusion of additional litters may have strengthened a marginal beneficial effect of 2'-FL. After the first $5 \mathrm{~d}$, there was no evidence of effects on small intestinal growth, permeability, digestive function or nutrient absorption. The FISH analyses revealed that bacteria were closely associated with the small intestinal mucosa, and dense bacterial colonisation of the enterocyte cytosol was also shown. This colonisation was not altered by dietary 2'-FL supplementation and did not correlate with endogenous $\alpha 1,2$ '-fucose or NEC lesions. The bacterial community that inhabited the intestinal lumen and mucosa was not different between 2'-FL and Control pigs, except that the relative abundance of Enterococcus was higher in 2'-FL pigs. No members of Bifidobacteria were detected by pyrosequencing, and it is possible that the previously reported bifidogenic effect of 2'-FL may be less relevant for pigs, as Bifidobacteria are not a major genus in the porcine gut ${ }^{(34)}$. 
An important consideration is whether the effects of HMO and 2'-FL are species specific. The limited effects of dietary 2'-FL on bacterial colonisation in preterm pigs are in contrast with previous studies in infants ${ }^{(8-11,13,14,35)}$ and in neonatal rats, where a di-sialylated HMO decreased NEC severity ${ }^{(18)}$. In both rat and cow's milk, sialylated HMO dominate, whereas porcine milk contains more fucosylated compounds, as in human milk, although the concentrations are lower and 2'-FL is not detected $^{(36)}$. Hence, we cannot exclude that dietary 2'-FL is less important for pigs, relative to humans, and that high endogenous levels of $\alpha 1,2^{\prime}$-fucose in the pig intestine from birth make supply of 2'-FL via the enteral milk diet less critical. On the other hand, it is also possible that the immature intestinal structure, function, microbiology and immunology in the newborn period make the effects of dietary 2'-FL less pronounced at this time, both for preterm and term birth, and across the different mammalian species

Preterm pigs are born with a highly immature intestine, resulting in spontaneous development of NEC lesions in a large proportion of pigs following bacterial colonisation and formula feeding ${ }^{(24)}$. This makes the preterm pig a highly sensitive model to investigate the mucosal responses to diet and microbial factors in preterm infants. In the present study, NEC lesion scores were relatively mild compared with some of our previous studies, but this cannot explain that 2'-FL failed to reduce NEC lesions and improve intestinal structure and function. Even if we only compare the severe NEC cases (NEC scores 4-6), there were no significant differences between 2'-FL and Control pigs (38 v. 47\%). As in neonatal intensive care units, NEC incidence varies over time and is determined by many factors other than diet and microbiota - for example, gestational age at birth, host genetics and other co-morbidities associated with preterm birth. In preterm pigs, NEC develops relatively early after birth and with a higher frequency compared with preterm infants, and early interventions may therefore have higher effect in pigs relative to infants. On the other hand, it is possible that the mild subclinical NEC-like lesions that are frequently observed in preterm pigs at autopsy (especially in the colon) ${ }^{(37)}$ may also be present temporarily in many infants that are never diagnosed with fulminant clinical NEC (most often affecting the small intestine). We conclude that addition of 2'-FL is unlikely to prevent the detrimental effects of formula-feeding during the 1st week after birth in preterm newborns. More studies on the species, age and diet dependency of HMO are required before the addition of 2'-FL or other HMO can be recommended for highly sensitive newborns.

\section{Acknowledgements}

The authors thank Mette Schmidt, Elin Skytte, Stine O. Rasmussen and Mette Maibom for assistance with animal studies and Annie Pedersen, Sophia Rasmussen and Kristina Møller for laboratory assistance.

The study was supported by The Danish Research Councils (0602-01613B, 0603-00774B)

M. S. C., S. B. B., M. V. Ø., M. L. J., D. S. N. and P. T. S. contributed to the design of the study. M. S. C., S. B. B., M. V. Ø.,
M. L. J. and P. T. S. performed the animal studies and laboratory analyses. M. S. C., S. B. B., M. V. Ø. and L. K. performed the data analyses, and finally all the authors contributed to the writing of the manuscript.

D. S. N. is affiliated with Glycosyn, the commercial partner donating 2'-fucosyllactose for the study. Other authors have no conflicts of interest.

\section{References}

1. Arslanoglu S, Corpeleijn W, Moro G, et al. (2013) Donor human milk for preterm infants: current evidence and research directions. I Pediatr Gastroenterol Nutr 57, $535-542$.

2. Bode L (2012) Human milk oligosaccharides: every baby needs a sugar mama. Glycobiology 22, 1147-1162.

3. Gabrielli O, Zampini L, Galeazzi T, et al. (2011) Preterm milk oligosaccharides during the first month of lactation. Pediatrics 128, e1520-e1531.

4. Newburg DS (2009) Neonatal protection by an innate immune system of human milk consisting of oligosaccharides and glycans. J Anim Sci 87, 26-34.

5. Gnoth MJ, Kunz C, Kinne-Saffran E, et al. (2000) Human milk oligosaccharides are minimally digested in vitro. J Nutr $\mathbf{1 3 0}$, 3014-3020.

6. Chaturvedi P, Warren CD, Altaye M, et al. (2001) Fucosylated human milk oligosaccharides vary between individuals and over the course of lactation. Glycobiology 11, 365-372.

7. Coppa GV, Gabrielli O, Zampini L, et al. (2011) Oligosaccharides in 4 different milk groups, Bifidobacteria, and Ruminococcus obeum. J Pediatr Gastroenterol Nutr $\mathbf{5 3}$, $80-87$.

8. Yu Z-T, Chen C, Kling DE, et al. (2013) The principal fucosylated oligosaccharides of human milk exhibit prebiotic properties on cultured infant microbiota. Glycobiology 23, 169-177.

9. Yu Z-T, Chen C \& Newburg DS (2013) Utilization of major fucosylated and sialylated human milk oligosaccharides by isolated human gut microbes. Glycobiology 23, 1281-1292.

10. Frydendahl K, Jensen TK, Andersen JS, et al. (2003) Association between the porcine Escherichia coli F18 receptor genotype and phenotype and susceptibility to colonisation and postweaning diarrhoea caused by E. coli O138:F18. Vet Microbiol 93, 39-51.

11. Coddens a, Verdonck F, Tiels P, et al. (2007) The agedependent expression of the F18 + E. coli receptor on porcine gut epithelial cells is positively correlated with the presence of histo-blood group antigens. Vet Microbiol 122, 332-341.

12. Weichert S, Jennewein S, Hüfner E, et al. (2013) Bioengineered 2'-fucosyllactose and 3-fucosyllactose inhibit the adhesion of Pseudomonas aeruginosa and enteric pathogens to human intestinal and respiratory cell lines. Nutr Res 33, 831-838.

13. Lane JA, Mehra RK, Carrington SD, et al. (2010) The food glycome: a source of protection against pathogen colonization in the gastrointestinal tract. Int J Food Microbiol 142, 1-13.

14. Ruiz-Palacios GM, Cervantes LE, Ramos P, et al. (2003) Campylobacter jejuni binds intestinal $\mathrm{H}(\mathrm{O})$ antigen (Fuc alpha 1, 2Gal beta 1, 4GlcNAc), and fucosyloligosaccharides of human milk inhibit its binding and infection. J Biol Chem 278, 14112-14120.

15. Morrow AL, Ruiz-Palacios GM, Altaye M, et al. (2004) Human milk oligosaccharides are associated with protection against diarrhea in breast-fed infants. J Pediatr 145, 297-303. 
16. Morowitz MJ, Poroyko V, Caplan M, et al. (2010) Redefining the role of intestinal microbes in the pathogenesis of necrotizing enterocolitis. Pediatrics 125, 777-785.

17. Morrow AL, Meinzen-Derr J, Huang P, et al. (2011) Fucosyltransferase 2 non-secretor and low secretor status predicts severe outcomes in premature infants. J Pediatr 158, 745-751.

18. Jantscher-Krenn E, Zherebtsov M, Nissan C, et al. (2012) The human milk oligosaccharide disialyllacto-N-tetraose prevents necrotising enterocolitis in neonatal rats. Gut 61, 1417-1425.

19. Van Niekerk E, Autran CA, Nel DG, et al. (2014) Milk oligosaccharides differ between HIV-Infected and HIV-uninfected mothers and are related to necrotizing enterocolitis incidence in their preterm very-low-birth-weight infants. $J$ Nutr $\mathbf{1 4 4}$ $1227-1233$.

20. Kuntz S, Rudloff S \& Kunz C (2008) Oligosaccharides from human milk influence growth-related characteristics of intestinally transformed and non-transformed intestinal cells. Br J Nutr 99, 462-471.

21. He Y, Liu S, Leone S, et al. (2014) Human colostrum oligosaccharides modulate major immunologic pathways of immature human intestine. Mucosal Immunol 7, 1326-1339.

22. He Y, Liu S, Kling DE, et al. (2016) The human milk oligosaccharide 2'-fucosyllactose modulates CD14 expression in human enterocytes, thereby attenuating LPS-induced inflammation. Gut 65, 33-46.

23. Sangild PT, Siggers RH, Schmidt M, et al. (2006) Diet- and colonization-dependent intestinal dysfunction predisposes to necrotizing enterocolitis in preterm pigs. Gastroenterology 130, 1776-1792.

24. Sangild PT, Thymann T, Schmidt M, et al. (2013) Invited review: the preterm pig as a model in pediatric gastroenterology. J Anim Sci 91, 4713-4729.

25. Buddington RK, Bering SB, Thymann T, et al. (2008) Aldohexose malabsorption in preterm pigs is directly related to the severity of necrotizing enterocolitis. Pediatr Res $\mathbf{6 3}, 382-387$.

26. Li Y, Østergaard MV, Jiang P, et al. (2013) Whey protein processing influences formula-induced gut maturation in preterm pigs. J Nutr 143, 1-9.

27. Østergaard MV, Bering SB, Jensen ML, et al. (2013) Modulation of intestinal inflammation by minimal enteral nutrition with amniotic fluid in preterm pigs. JPEN J Parenter Enteral Nutr 38, 576-586.

28. Bangsgaard Bendtsen KM, Krych L, Sørensen DB, et al. (2012) Gut microbiota composition is correlated to grid floor induced stress and behavior in the BALB/c mouse. PLOS ONE $\mathbf{7}$, e46231.

29. Mølbak L, Thomsen LE, Jensen TK, et al. (2007) Increased amount of Bifidobacterium thermacidophilum and Megasphaera elsdenii in the colonic microbiota of pigs fed a swine dysentery preventive diet containing chicory roots and sweet lupine. J Appl Microbiol 103, 1853-1867.

30. Thymann T, Møller KH, Stoll B, et al. (2009) Carbohydrate maldigestion induces necrotizing enterocolitis in preterm pigs. Am I Physiol Gastrointest Liver Physiol 297. G1115-G1125

31. Jensen ML, Cilieborg MS, Thymann T, et al. (2014) Antibiotics modulate intestinal immunity and prevent necrotizing enterocolitis in preterm neonatal piglets. Am J Physiol Gastrointest Liver Physiol 306, G59-G71.

32. Newburg DS, Ruiz-Palacios GM \& Morrow AL (2005) Human milk glycans protect infants against enteric pathogens. Annu Rev Nutr 25, 37-58.

33. Cilieborg MS, Sangild PT, Jensen ML, et al. (2016) $\alpha 1,2-$ Fucosyllactose does not improve intestinal function or prevent Escherichia coli F18 diarrhea in newborn pigs. J Pediatr Gastroenterol Nutr (Epublication ahead of print version 27 May 2016).

34. Heinritz SN, Mosenthin R \& Weiss E (2013) Use of pigs as a potential model for research into dietary modulation of the human gut microbiota. Nutr Res Rev $\mathbf{2 6}$, 191-209.

35. Coppa GV, Zampini L, Galeazzi T, et al. (2006) Human milk oligosaccharides inhibit the adhesion to Caco-2 cells of diarrheal pathogens: Escherichia coli, Vibrio cholerae, and Salmonella fyris. Pediatr Res 59, 377-382.

36. Tao N, Ochonicky KL, German JB, et al. (2010) Structural determination and daily variations of porcine milk oligosaccharides. J Agric Food Chem 58, 4653-4659.

37. Cao M, Andersen AD, Li Y, et al. (2016) Physical activity and gastric residuals as biomarkers for region-specific NEC lesions in preterm neonates. Neonatology 110, 241-247. 